\title{
Does ambient light at night reduce total melatonin production?
}

\author{
Christopher C.M. Kyba, ${ }^{1,2}$ Thomas Kantermann ${ }^{3,4}$
}

\begin{abstract}
${ }^{1}$ Deutsches GeoForschungsZentrum GFZ, ${ }^{2}$ Leibniz-Institute of Freshwater Ecology and Inland Fisheries, ${ }^{3}$ Chronobiology Unit, Groningen Institute for Evolutionary Life Sciences, University of Groningen, Groningen, The Netherlands, ${ }^{4}$ Institute for Occupational, Social and Environmental Medicine, Clinical Centre Ludwig-Maximilians University Munich, Munich, Germany
\end{abstract}

Dear Editor,

It was with great interest that we read the recent study by Hersh et $\mathrm{al}^{1}$ on the effects of sleep and light at night on melatonin in adolescents. Of particular interest was their focus on electronic use after "lights out". The authors highlight the importance of understanding what effects this may have on sleep, citing a survey that showed that $72 \%$ of American 13-18 year olds regularly use a cellphone or computer before trying to go to sleep. ${ }^{2}$ In their study, Hersh et al ${ }^{1}$ did not observe a significant suppression in urinary morning melatonin (aMT6s) levels with respect to the use of electronic devices between lights off and sleep onset. Therefore, the authors conclude that "nighttime behaviors of adolescents by and large do not impact urinary melatonin levels". Absence of evidence, however, is not the same as evidence of absence, and we believe that the authors' conclusion is premature.

Key words: Chronotype, Light at Night; Melatonin

\section{Address for correspondence:}

Christopher Kyba, Telegraphenberg, 14473 Potsdam, Germany;

Tel.: +49 331288 28973, Fax: +49 3312881192 ,

E-mail:kyba@gfz-potsdam.de

Received: 22-07-2015, Accepted: 27-07-2015
A casual reader not examining their tables may be surprised to learn that the data of Hersh et $\mathrm{al}^{1}$ in fact are most consistent with a relatively large melatonin reduction associated with the use of electronic devices. Compared to adolescents who used electronic devices for less than one hour after lights off on weekdays, the authors observed a mean reduction in aMT6s of $21 \%$ in adolescents who used electronics for $1-2$ hours during the same period. Their findings, therefore, are entirely consistent with two opposing hypotheses: First, that electronic use has no effect on melatonin, and second that electronic media are associated with a relatively large decrease in aMT6s. Presenting the conclusions in this way makes a much stronger case for the need for a follow-up study, with an approximately 15 times larger sample size.

Hersh et $\mathrm{al}^{1}$ also demonstrated that subjective assessments of ambient bedroom lighting was poorly correlated with aMT6s. However, the data suggest that such subjective assessments may not be accurate: only 5 participants reported being able to read, despite the fact that 22 said a light or TV was on in or near the bedroom overnight and 45 reported a light outside of the bedroom window. We suggest followup studies should add (freely available) nighttime imagery of the participant's location ${ }^{3}$ to the study repertoire, combined with questions about the use of sleep masks and/or window shades, and importantly, light exposure history the previous day. ${ }^{4}$ Chronotype, as the authors point out, is essential to disentangle 
the individual contribution of light exposure on the circadian system. ${ }^{5}$ However, when chronotype is to be used as a reference measure for circadian phase, it is preferable to consider other individual phase markers in addition to using amplitude measures (such as aMT6s here). One example is the dim light melatonin onset, which is highly correlated with chronotype. ${ }^{6}$

As a final thought, it is worth considering the role of smartphones in future studies. ${ }^{7}$ The iPhone camera, for example, can accurately measure radiance down to starlight levels, ${ }^{8}$ and the gyroscope and accelerometer provide evidence of the participant's activity. Together, these sensors could provide far more accurate assessment of "in bed", "lights out", and "phone off" timing than participant reporting.

\section{FINANCIAL SUPPORT}

CCMK is financed by the GFZ German Research Centre for Geosciences. TK is supported by the Technology Foundation STW grant P10-18/12186. CCMK and TK acknowledge the support of EU COST Action ES 1204 for providing the opportunity for in-person discussions.

\section{REFERENCES}

1. Hersh C, Sisti J, Richiutti V, Schernhammer E, 2015 The effects of sleep and light at night on melatonin in adolescents. Hormones (Athens) 14: 399-409.

2. National Sleep Foundation, 2011 Sleep in America Poll: Communications Technology in the Bedroom. http://www.sleepfoundation.org/article/sleep-americapolls/2011-communications-technology-use-and-sleep

3. Kyba CCM, Aronson DJ, 2015 Assessing exposure to outdoor lighting and heath risks. Epidemiology 26: e50.

4. Hebert M, Martin SK, Lee C, Eastman CI, 2002 The effects of prior light history on the suppression of melatonin by light in humans. J Pineal Res 33: 198203.

5. Kantermann T, Roenneberg T, 2009 Is light-at-night a health risk factor or a health risk predictor? Chronobiol Int 26: 1069-1074.

6. Kantermann T, Sung H, Burgess HJ, Comparing the Morningness-Eveningness Questionnaire and Munich ChronoType Questionnaire to the Dim Light Melatonin Onset. J Biol Rhythms: in press.

7. Wahl F, Kantermann T, Amft O, 2014 How much light do you get? Estimating daily light exposure using smartphones. Proceedings of the 2014 ACM International Symposium on Wearable Computers.

8. Dark Sky Meter App: www.darkskymeter.com 